\title{
Biliary complication in living donor liver transplantation in single center, experience
}

\author{
Eunkyoung Jwa ${ }^{1}$, Joo Dong Kim² ${ }^{2}$ Dong Lak Choi ${ }^{2}$ \\ 1Department of Surgery-Transplantation, Asan Medical Center, University of Ulsan College of Medicine, Seoul, Korea \\ ${ }^{2}$ Department of Surgery-Transplantation, Daegu Catholic University Medical Center, Daegu, Korea
}

Background: A shortage of deceased donors has resulted in living donor liver transplantation (LDLT) becoming a major treatment strategy for end-stage liver disease, including hepatocellular carcinoma. LDLT has been accepted as an established treatment modality. Biliary complication (BC) is an Achilles in LDLT. The way to decrease BC is still being debated. The Daegu Catholic Medical Center (DCMC) review 2-year data for BC and evaluated risk factor to find the way to improved biliary complications.

Methods: From January 1, 2015, to December 31, 2016, 126 liver transplants were performed in DCMC. One hundred and six patients were performed living donor liver transplantation and 20 patients were performed deceased donor liver transplantation. There were no $B C$ in patients with deceased donor liver transplantation (DDLT).

Results: For 2 years, 126 patient received LT (LDLT, 106; DDLT, 20). Among 106 cases of LDLT, a total of 19 patients suffered from BC (17.9\%). Two patients suffered from bile leak and 16 patients suffered from biliary stricture and one patient suffered from both leakage and stricture. Compared to the $\mathrm{BC}$ group and non-BC groups, only ABO incomplete factor was significant. Interestingly, duct anastomosis, stent types, and duct size were not significant risk factors. The BC groups show lower 5 -year graft survival than non-BC group but was not significant.

Conclusions: Still, BC incidence is major complication in LDLT. We must put effort to reduce BC. We should try prospective study design to decrease BC.

Corresponding author: Eunkyoung Jwa

E-mail:jjwa03@gmail.com

(c) The Korean Society for Transplantation

This is an Open Access article distributed under the terms of the Creative Commons Attribution Non-Commercial License (http://creativecommons.org/licenses/by-nc/4.0/) which permits unrestricted non-commercial use, distribution, and reproduction in any medium, provided the original work is properly cited. 